\title{
Continuation semantics for multi-quantifier sentences: operation-based approaches
}

\author{
Justyna Grudzińska and Marek Zawadowski
}

November 2, 2018

\begin{abstract}
Classical scope-assignment strategies for multi-quantifier sentences involve quantifier phrase (QP)-movement (e.g., [13, [14]). More recent continuation-based approaches provide a compelling alternative, for they interpret QP's in situ - without resorting to Logical Forms or any structures beyond the overt syntax. The continuation-based strategies can be divided into two groups: those that locate the source of scope-ambiguity in the rules of semantic composition (e.g., [1]) and those that attribute it to the lexical entries for the quantifier words (e.g., 9, 2]). In this paper, we focus on the former operation-based approaches and the nature of the semantic operations involved. More specifically, we discuss three such possible operation-based strategies for multi-quantifier sentences, together with their relative merits and costs.
\end{abstract}

\section{Introduction}

Multi-quantifier sentences have been known to be ambiguous with different readings corresponding to how various quantifier phrases (QPs) are semantically related in the sentence. For example,

(1) Some teacher gave every student most books

admits of six different readings, and in general a simple sentence with $n$ QPs will be (at least) $n$ ! ways ambiguous (we only consider readings where QPs are linearly ordered - what we will call asymmetric readings).

Sentence (1) can be represented as a Syntactic (Surface Structure) Tree and the challenge is to obtain corresponding Semantic (Computation) Trees that compute the truth-value of the sentence in each of its readings 

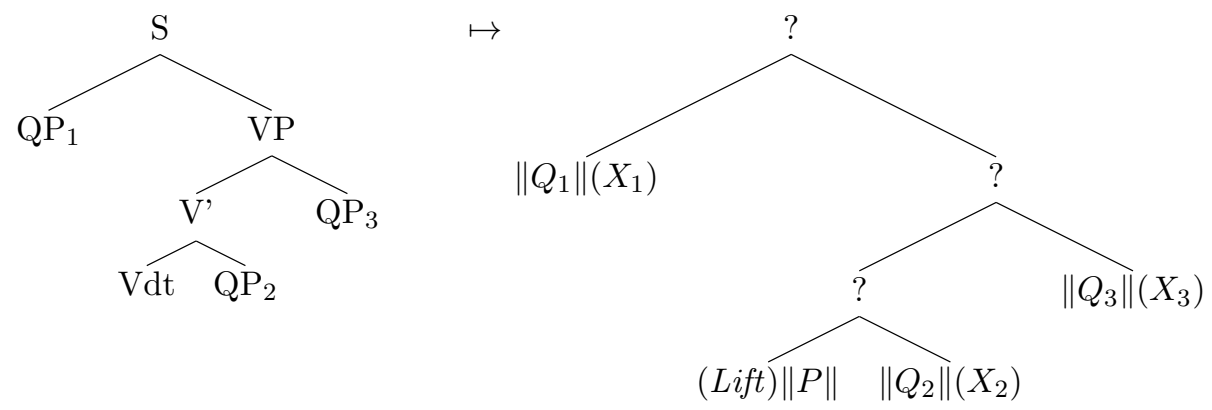

We think here of Computation Trees by analogy with mathematical expressions, e.g.

$$
((2-7)-8)+((12+5): 7)
$$

can be represented as

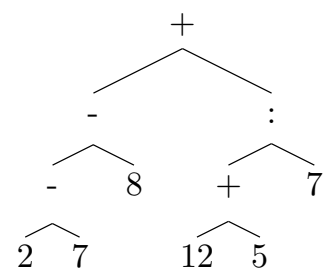

i.e. a labeled binary tree where the leaves of this tree are labeled with values and the internal nodes are labeled with operations that will be applied in the computation to the values obtained from the computations of the left and right subtrees.

The process transforming Surface Structure Trees into the Semantic (Computation) Trees has been variously implemented in linguistics. Below we provide a list of desiderata relevant for the variety of the continuationbased semantics to be discussed in this paper.

(1) The semantics should be empirically adequate, i.e., it should allow us to calculate the truth-value of a given sentence in each of its readings.

(2) The semantics should be in situ, i.e., Computation Trees should have the same shape as Surface Structure Trees (with leaves labeled with interpretations of lexical items and inner nodes labeled with semantic operations).

(3) The semantic operations used should be kept as simple as possible.

(4) The semantic operations used should be kept as uniform as possible.

(5) The interpretation process should operate 'on the fly', i.e., the particular reading(s) of a sentence should be determined as late as possible.

Classical movement analyses (involving quantifier phrase (QP)-movement, e.g., [13, 14]) generally meet desiderata (1), (3) and (4). More recent continuation-based approaches provide a compelling alternative, for they interpret QP's in situ - without resorting to Logical Forms or any structures 
beyond the overt syntax. They achieve this at a certain price though (including loss in simplicity/uniformity of the semantic operations used) - as summarized by the table below

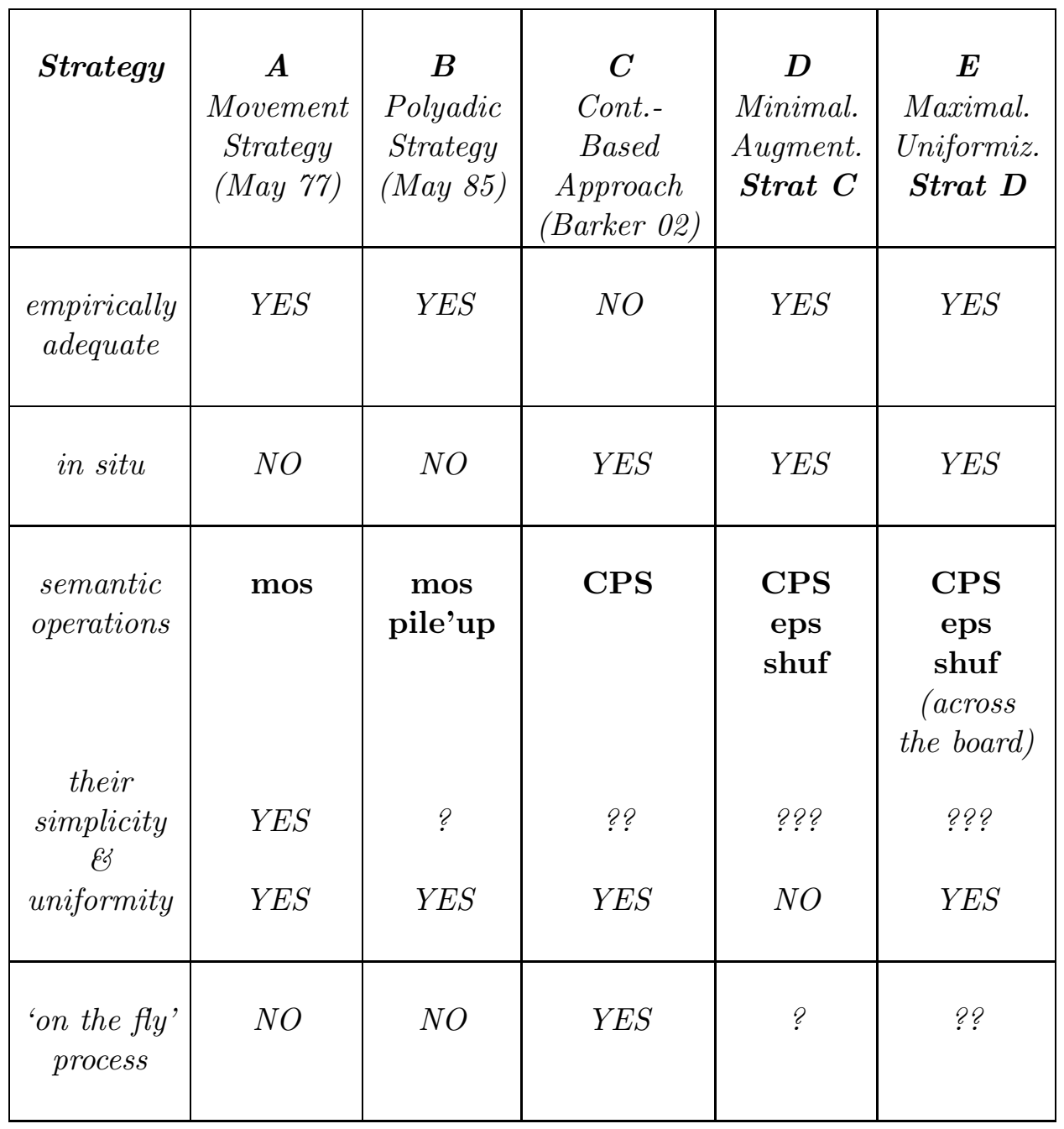

In this paper, we first briefly recall the definitions of the semantic operations used in strategies A, B and C, i.e., mos'es, pile'up'es, CPS'es (for the details, see [20]). We then discuss the three in situ strategies $\mathbf{C}, \mathbf{D}$ and $\mathbf{E}$, together with their relative merits and costs with respect to the desiderata introduced. 


\section{Semantic Operations}

As noticed in [1] and [7, a generalized quantifier on a set $X$ is an element of $\mathcal{C}(X)$, the value of the continuation monad $\mathcal{C}$ on $X$. Continuation-based semantics make heavy use of the computational machinery connected to the monad $\mathcal{C}$ (its strength and derived operations). Here we only recall the definitions of the continuation monad, strengths and derived operations. For more details, we refer the reader to [20].

\subsection{Continuation Monad and Strengths}

We shall be working in the (cartesian closed) category of sets Set. The category Set has sets as objects. A morphism in Set from an object (set) X to an object (set) $\mathrm{Y}$ is a function $f: X \rightarrow Y$ from $X$ to $Y$. For the general notion of a strong monad, see the appendix.

\section{Continuation monad $\mathcal{C}$ - endofunctor}

- At the level of objects, it is just twice iterated power-set construction, i.e. for set $X, \mathcal{C}(X)=\mathcal{P}^{2}(X)$. $(\mathbf{t}=\{0,1\}, \mathcal{P}(X)=X \Rightarrow \mathbf{t}-$ powerset of $X)$

- At the level of morphisms, it is an inverse image of an inverse image, i.e., function $f: X \rightarrow Y$ induces an inverse image function between powersets

$$
\begin{gathered}
\mathcal{P}(f)=f^{-1}: \mathcal{P}(Y) \rightarrow \mathcal{P}(X) \\
h \mapsto h \circ f, \quad \mathcal{P}(f)=\lambda h_{: \mathcal{P}(Y)} \cdot \lambda x_{: X} \cdot h(f x)
\end{gathered}
$$

Taking again an inverse image function, we have

$$
\begin{aligned}
& \mathcal{C}(f)=\mathcal{P}\left(f^{-1}\right): \mathcal{C}(X)=\mathcal{P}^{2}(X) \rightarrow \mathcal{P}^{2}(Y)=\mathcal{C}(Y) \\
& Q \mapsto Q \circ f^{-1}, \mathcal{C}(f)(Q)=\lambda h_{: \mathcal{P}(Y)} \cdot Q\left(\lambda x_{: X} \cdot h(f x)\right)
\end{aligned}
$$

for $Q \in \mathcal{C}(X)$.

\section{Continuation monad $\mathcal{C}$ - natural transformations}

- The unit

$$
\eta_{X}: X \rightarrow \mathcal{C}(X)
$$

is given by

$$
\eta_{X}(x)=\lambda h_{: \mathcal{P}(X)} \cdot h(x)
$$

for $x \in X$.

(lifts elements of $X$ as $\mathcal{C}$-computations.) 
- The multiplication

$$
\mu_{X}: \mathcal{C}^{2}(X) \longrightarrow \mathcal{C}(X)
$$

is given by

$$
\mu_{X}(\mathcal{F})(h)=\mathcal{F}\left(\lambda D_{: \mathcal{C}(X)} \cdot D(h)\right)
$$

for $\mathcal{F} \in \mathcal{C}^{2}(X)$ and $h \in \mathcal{P}(X)$.

(flattens $\mathcal{C}$-computations on $\mathcal{C}$-computations to $\mathcal{C}$-computations.)

\section{Continuation monad $\mathcal{C}$ - strengths}

For the continuation monad, the left strength is

$$
\begin{gathered}
\mathbf{s t}^{l}: \mathcal{C}(X) \times Y \longrightarrow \mathcal{C}(X \times Y) \\
\mathbf{s t}^{l}(N, y)=\lambda c_{: \mathcal{P}(X \times Y)} \cdot N\left(\lambda x_{: X} \cdot c(x, y)\right)
\end{gathered}
$$

for $N \in \mathcal{C}(X)$ and $y \in Y$, and the right strength is

$$
\begin{gathered}
\mathbf{s t}^{r}: X \times \mathcal{C}(Y) \longrightarrow \mathcal{C}(X \times Y) \\
\text { st }^{r}(x, M)=\lambda c_{: \mathcal{P}(X \times Y)} \cdot M\left(\lambda y_{: Y} \cdot c(x, y)\right)
\end{gathered}
$$

for $x \in X$ and $M \in \mathcal{C}(Y)$.

Strengths allow to lift pairs of $\mathcal{C}$-computations to $\mathcal{C}$-computations on products.

\subsection{Derived Operations}

\section{pile'up operations}

Using both strengths, we can define pile' up-operations.

For $M \in \mathcal{C}(X)$ and $N \in \mathcal{C}(Y)$, we have

$$
\begin{aligned}
& \text { pile'up }^{l}: \mathcal{C}(X) \times \mathcal{C}(Y) \rightarrow \mathcal{C}(X \times Y) \\
& \operatorname{pile}^{\prime} \mathbf{u p}^{l}(M, N)=\lambda c_{: \mathcal{P}(X \times Y)} \cdot M\left(\lambda x_{: X} \cdot N\left(\lambda y_{: Y} c(x, y)\right)\right.
\end{aligned}
$$

and

$$
\begin{aligned}
& \text { pile' }^{\prime} \mathbf{u p}^{r}: \mathcal{C}(X) \times \mathcal{C}(Y) \rightarrow \mathcal{C}(X \times Y) . \\
& \text { pile }^{\prime} \mathbf{u p}^{r}(M, N)=\lambda c_{: \mathcal{P}}(X \times Y) \cdot N\left(\lambda y: Y \cdot M\left(\lambda x_{: X} c(x, y)\right) .\right.
\end{aligned}
$$

Thus pile'up-operations put (interpretations of) quantifiers in order, either first before the second or the second before the first. 


\section{CPS operations}

Now, we can define CPS-transforms. For $f: X \times Y \rightarrow Z$, we have

$$
\mathbf{C P S}^{l}(f)=\mathcal{C}(f) \circ \operatorname{pile}^{\prime} \mathbf{u p}_{X, Y}^{l}: \mathcal{C}(X) \times \mathcal{C}(Y) \longrightarrow \mathcal{C}(Z)
$$

given, for $M \in \mathcal{C}(X)$ and $N \in \mathcal{C}(Y)$, by

$$
\operatorname{CPS}^{l}(f)(M, N)=\lambda h_{: \mathcal{P}(Z)} \cdot M\left(\lambda x_{: X} \cdot N\left(\lambda y_{: Y} \cdot h(f(x, y))\right)\right) .
$$

Right version is similar.

The most popular CPS-transforms are those for the evaluation (application) $e v: X \times(X \Rightarrow Y) \rightarrow Y$

$$
\operatorname{CPS}^{l}(e v)=\mathcal{C}(e v) \circ \text { pile' }^{\prime} \mathbf{u p}_{X, X \Rightarrow Y}^{l}: \mathcal{C}(X) \times \mathcal{C}(X \Rightarrow Y) \longrightarrow \mathcal{C}(Y)
$$

given, for $M \in \mathcal{C}(X)$ and $N \in \mathcal{C}(X \Rightarrow Y)$, by

$$
\operatorname{CPS}^{l}(e v)(M, N)=\lambda h_{: \mathcal{P}(Y)} \cdot M\left(\lambda x_{: X} \cdot N\left(\lambda g_{: X \Rightarrow Y} \cdot h(g x)\right)\right) .
$$

Right version is similar.

\section{Some functions having products as their domains}

There are also other morphisms having useful transforms. Below we list some to introduce notation.

Left evaluations

$$
\begin{gathered}
\operatorname{eps}_{X}^{l}=\lambda h_{: \mathcal{P}(X)} \cdot \lambda x_{: X} \cdot h(x): \mathcal{P}(X) \times X \rightarrow \mathbf{t} \\
\operatorname{eps}_{Y}^{l}{ }_{Y}^{,}=\operatorname{eps}_{Y}^{l}=\lambda c_{: \mathcal{P}(X \times Y)} \cdot \lambda y_{: Y} \cdot \lambda x_{: X} \cdot c(x, y): \mathcal{P}(X \times Y) \times Y \rightarrow \mathcal{P}(X) ;
\end{gathered}
$$

and right evaluations

$$
\begin{gathered}
\operatorname{eps}_{X}^{r}=\lambda x_{: X} \cdot \lambda h_{: \mathcal{P}(X)} \cdot h(x): X \times \mathcal{P}(X) \rightarrow \mathbf{t} \\
\operatorname{eps}_{Y}^{r, X}=\operatorname{eps}_{Y}^{r}=\lambda y_{Y} \cdot \lambda c_{: \mathcal{P}(X \times Y)} \cdot \lambda x_{: X} \cdot c(x, y): Y \times \mathcal{P}(X \times Y) \rightarrow \mathcal{P}(X) .
\end{gathered}
$$

mos-operations are the algebraic counterpart of the familiar interpretation of generalized quantifiers of Mostowski (again, we give definitions for total and partial case). Left mos'es

$$
\operatorname{mos}_{X}^{l}=\lambda Q_{: \mathcal{C}(X)} \cdot \lambda c_{: \mathcal{P}(X)} \cdot Q(c): \mathcal{C}(X) \times \mathcal{P}(X) \rightarrow \mathbf{t} ;
$$

$\operatorname{mos}_{Y}^{l}=\lambda Q_{: \mathcal{C}(Y)} \cdot \lambda c_{: \mathcal{P}(X \times Y)} \cdot \lambda x_{: X} \cdot Q\left(\lambda y_{: Y} \cdot c(x, y)\right): \mathcal{C}(Y) \times \mathcal{P}(X \times Y) \rightarrow \mathcal{P}(X) ;$

and right mos'es

$$
\begin{gathered}
\operatorname{mos}^{r}=\lambda c_{: \mathcal{P}(X)} \cdot \lambda Q_{: \mathcal{C}(X)} \cdot Q(c): \mathcal{P}(X) \times \mathcal{C}(X) \rightarrow \mathbf{t} \\
\operatorname{mos}_{Y}^{r}=\lambda c_{: \mathcal{P}(X \times Y)} \cdot \lambda Q_{: \mathcal{C}(Y)} \cdot \lambda x_{: X} \cdot Q\left(\lambda y_{: Y} \cdot c(x, y)\right): \mathcal{P}(X \times Y) \times \mathcal{C}(Y) \rightarrow \mathcal{P}(X) .
\end{gathered}
$$




\section{Continuation-Based In Situ Strategies}

Below we illustrate Strategy C, D \& E on examples involving one, two and three QPs (for the description of Strategies A \& B, see [20]). In each strategy, the leaves in the Computation Trees have the same labels: QPs are interpreted as $\mathcal{C}$-computations, and predicates are interpreted as lifted ('continuized') relations. The main difference among the three approaches consists in the operations (CPS'es, shuf'es, eps'es) used as labels of the inner nodes of the Computation Trees.

\subsection{Strategy C}

Sentence with one QP, e.g. Every kid (most kids) entered.

(C1) Surface Structure Tree and the corresponding Computation Tree

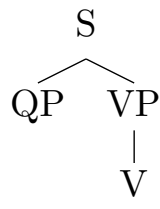

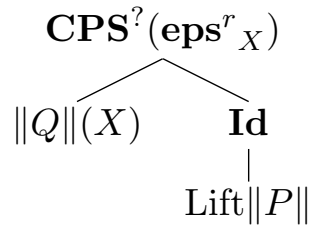

with Id standing for the identity operation. We use CPS ${ }^{\text {? }}$ when it does not matter whether we apply $\mathbf{C P S}^{l}$ or $\mathbf{C P S}^{r}$. This is the case when one of the arguments is a lifted element (like interpretations of predicates in this strategy). Strategy $\mathbf{C}$ yields one reading for a sentence with one QPs.

Sentence with two QPs, e.g. Every girl likes a boy.

(C2) Surface Structure Tree and the corresponding Computation Tree
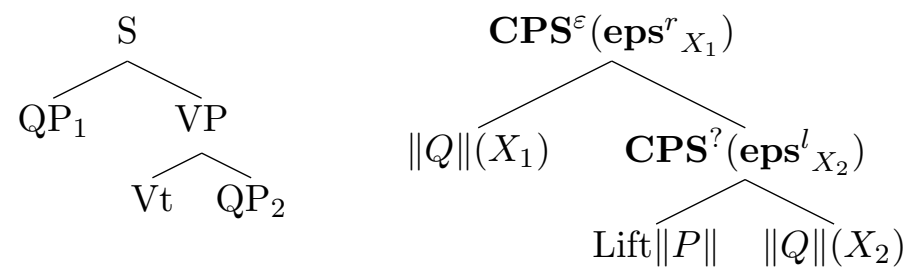

with $\varepsilon \in\{l, r\}$. Depending on whether we use $\mathbf{C P S}^{l}$ or $\mathbf{C P S}^{r}$, we get either one or the other of the two asymmetric readings for a sentence with two QPs. Thus Strategy C yields two readings for a sentence with two QPs, corresponding to the two CPS'es.

Sentence with three QPs, e.g. Some teacher gave every student most books. 
(C3) Surface Structure Tree and the corresponding Computation Tree
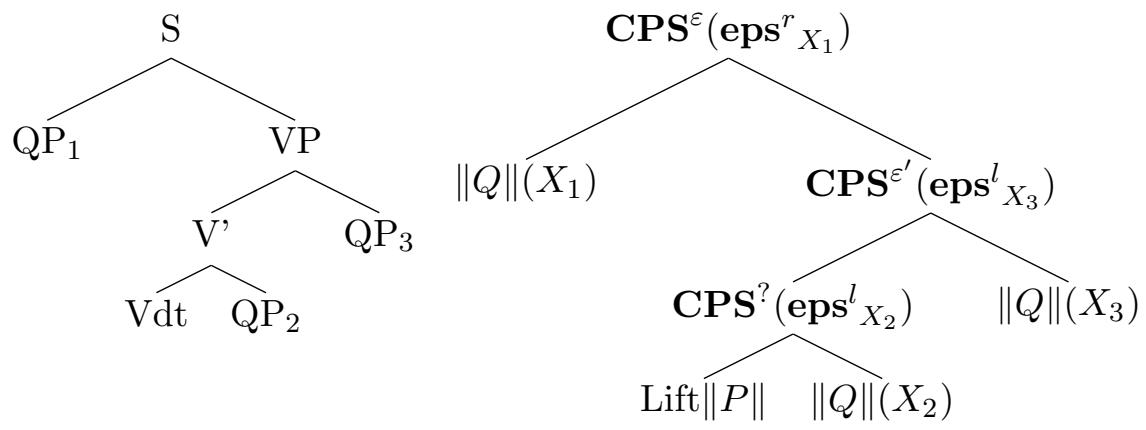

Strategy $\mathbf{C}$ provides four asymmetric readings for a sentence with a ditransitive verb such that QP in subject position can be placed either first or last only, corresponding to the four possible combinations of the two CPS'es.

Strategy C allows a uniform in situ analysis of quantifiers. However, it cannot be straightforwardly extended to account for sentences involving 3 QPs - as discussed in [4] and proved in [20], it only provides four out of six readings available for such sentences. Below we define Strategy D, a minimally augmented empirically adequate version of Strategy C.

\subsection{Strategy D}

To include the two readings missing from Strategy C, Strategy D combines CPS'es to define two new operations: shuf ${ }^{l}$ and shuf $^{r}$ (one can also combine pile'up- and CPS-operations to define those new operations, this is left for another place though).

Sentence with one QP, e.g. Every kid (most kids) entered.

(D1) Surface Structure Tree and the corresponding Computation Tree

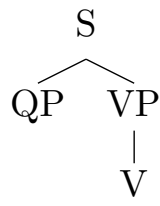

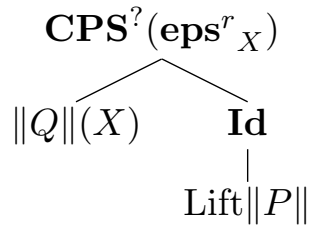

Just as in Strategy C, Strategy D yields one reading for a sentence with one QPs.

Sentence with two QPs, e.g. Every girl likes a boy. 
(D2) Surface Structure Tree and the corresponding Computation Tree
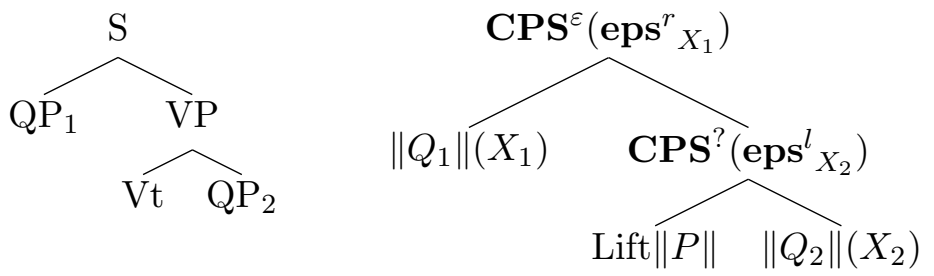

Just as in Strategy C, Strategy D yields both asymmetric readings for such sentences.

Sentence with three QPs, e.g. Some teacher gave every student most books.

(D3) Surface Structure Tree and the corresponding Computation Tree
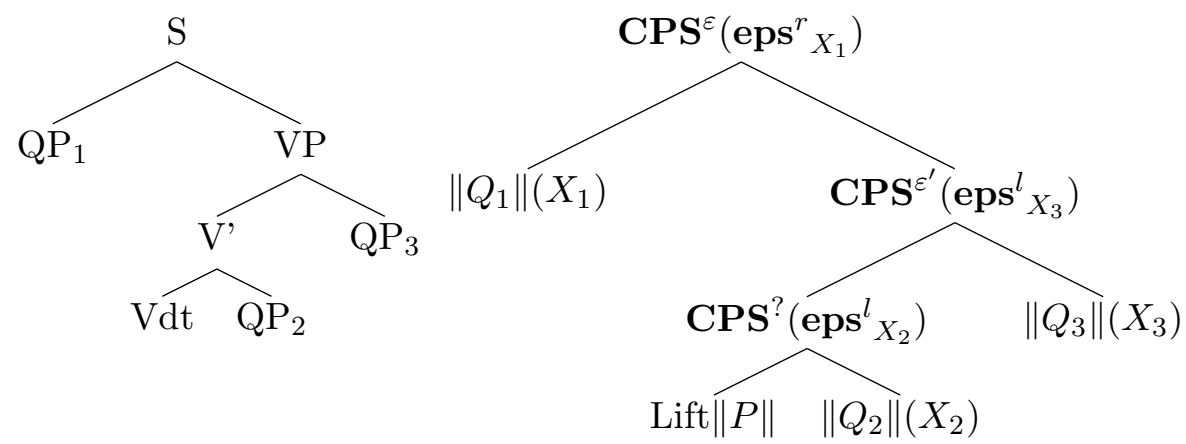

Just as in Strategy C, the Computation Tree above gives rise to the four asymmetric readings for sentences with ditransitive verbs such that QP in subject position can be placed either first or last only. To get the first missing reading

$Q P_{3}>Q P_{1}>Q P_{2}$

we define a new operation

$$
\operatorname{shuf}^{r}: \mathcal{C P}\left(X_{1} \times X_{3}\right) \times \mathcal{C}\left(X_{3}\right) \rightarrow \mathcal{P C}\left(X_{1}\right)
$$

such that

$\operatorname{shuf}^{r}\left(S_{2}, S_{3}\right)=\lambda S_{1: \mathcal{C}\left(X_{1}\right)} \cdot \mathbf{C P S}^{l}\left(\operatorname{eps}^{r} X_{3}\right)\left(S_{3}, \operatorname{CPS}^{l}\left(\operatorname{eps}^{r}{ }_{X_{1}}\right)\left(S_{1}, S_{2}\right)\right)\left(i d_{t}\right)$

for $S_{2} \in \mathcal{C P}\left(X_{1} \times X_{3}\right)$ and $S_{3} \in \mathcal{C}\left(X_{3}\right)$.

The corresponding computation tree is now as follows 
(D3') Computation Tree

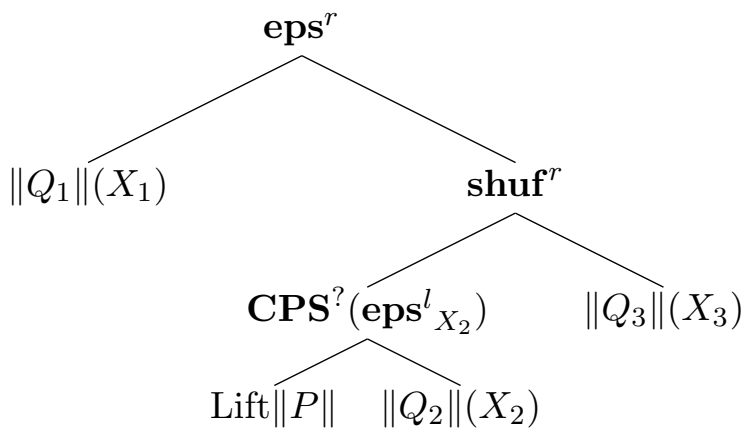

To get the second of the two missing readings

$Q P_{2}>Q P_{1}>Q P_{3}$,

we define a new operation

$$
\begin{aligned}
& \operatorname{shuf}^{l}: \mathcal{C P}\left(X_{1} \times X_{3}\right) \times \mathcal{C}\left(X_{3}\right) \rightarrow \mathcal{P C}\left(X_{1}\right) \\
& \operatorname{shuf}^{l}\left(S_{2}, S_{3}\right)=\lambda S_{1: \mathcal{C}\left(X_{1}\right)} \cdot \mathbf{C P S}^{l}\left(\mathbf{e p s}^{l} X_{3}\right)\left(\mathbf{C P S}^{l}\left(\mathbf{e p s}^{l}{ }_{X_{1}}\right)\left(S_{2}, S_{1}\right), S_{3}\right)\left(i d_{t}\right) \\
& \text { for } S_{2} \in \mathcal{C P}\left(X_{1} \times X_{3}\right) \text { and } S_{3} \in \mathcal{C}\left(X_{3}\right) .
\end{aligned}
$$

The corresponding computation tree is now as follows

(D3") Computation Tree

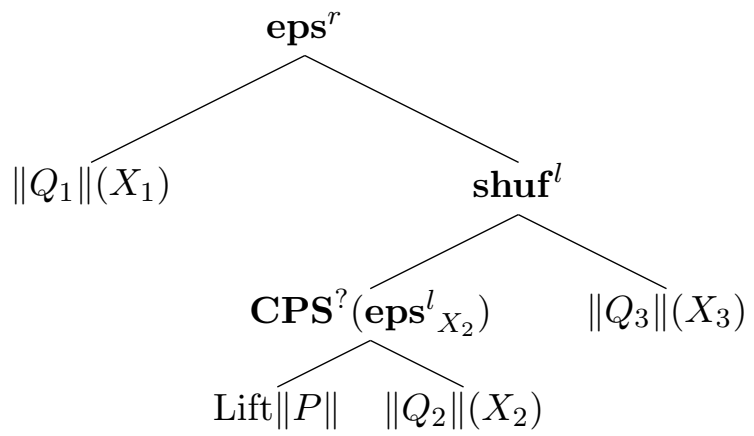

In Strategy $\mathbf{D}$, unlike in Strategy $\mathbf{C}$, we get all the asymmetric readings. Thus Strategy D is both in situ and free of the empirical deficiencies in Strategy C. This is achieved at the price, though, of extending the list of operations (adopted in strategy $\mathrm{C}$ ) by two more involved operations: left and right shuf. Moreover, the process translating Surface Structure Trees into the Semantic (Computation) Trees loses its uniformity - depending on the class of sentences (involving one, two or three QPs) and the particular readings considered, different semantic operations are used. Below we define Strategy E, a maximally uniformized version of Strategy D. 


\subsection{Strategy E}

In Strategy E, for each asymmetric reading its respective shuf-operation will be defined. We will index shuf'es with permutations of the $n$ distinct QPs involved, i.e., shuf ${ }^{\sigma}$ will denote the operation that determines the reading of the sentence with the QPs ordered according to the permutation

$\sigma$.

Sentence with one QP, e.g. Every kid (most kids) entered.

(E1) Surface Structure Tree and the corresponding Computation Tree
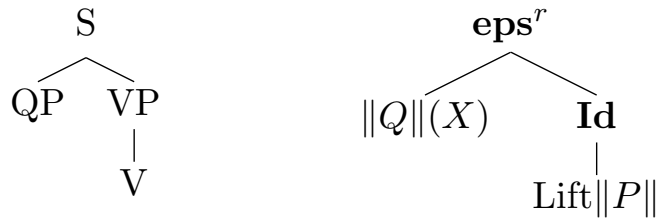

Strategy E yields one reading for a sentence with one QPs.

Sentence with two QPs, e.g. Every girl likes a boy.

(E2) Surface Structure Tree

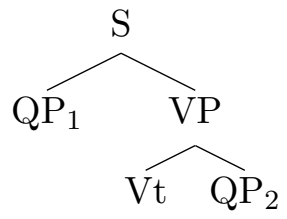

$Q P_{1}>Q P_{2}$

$$
\operatorname{shuf}^{1,2}: \mathcal{C P}\left(X_{1} \times X_{2}\right) \times \mathcal{C}\left(X_{2}\right) \rightarrow \mathcal{P C}\left(X_{1}\right)
$$

$\operatorname{shuf}^{1,2}\left(S, S_{2}\right)=\lambda S_{1: \mathcal{C}\left(X_{1}\right)} \cdot \mathbf{C P S}^{l}\left(\operatorname{eps}^{r}{ }_{X_{1}}\right)\left(S_{1}, \operatorname{CPS}^{l}\left(\operatorname{eps}_{X_{2}}^{l}\right)\left(S, S_{2}\right)\right)\left(i d_{t}\right)$

for $S \in \mathcal{C P}\left(X_{1} \times X_{2}\right)$ and $S_{2} \in \mathcal{C}\left(X_{2}\right)$.

$Q P_{2}>Q P_{1}$

$$
\operatorname{shuf}^{2,1}: \mathcal{C P}\left(X_{1} \times X_{2}\right) \times \mathcal{C}\left(X_{2}\right) \rightarrow \mathcal{P C}\left(X_{1}\right)
$$

$\operatorname{shuf}^{2,1}\left(S, S_{2}\right)=\lambda S_{1: \mathcal{C}\left(X_{1}\right)} \cdot \mathbf{C P S}^{l}\left(\operatorname{eps}^{l}{ }_{X_{1}}\right)\left(\operatorname{CPS}^{l}\left(\operatorname{eps}^{l}{ }_{X_{2}}\right)\left(S, S_{2}\right), S_{1}\right)\left(i d_{t}\right)$

for $S \in \mathcal{C P}\left(X_{1} \times X_{2}\right)$ and $S_{2} \in \mathcal{C}\left(X_{2}\right)$.

The corresponding computation trees are now as follows 


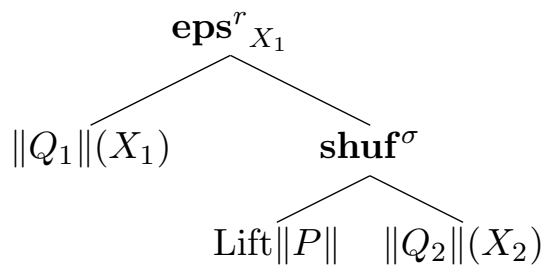

where $\sigma$ is a permutation of $\{1,2\}$.

Sentence with three QPs, e.g. Some teacher gave every student most books.

(E3) Surface Structure Tree

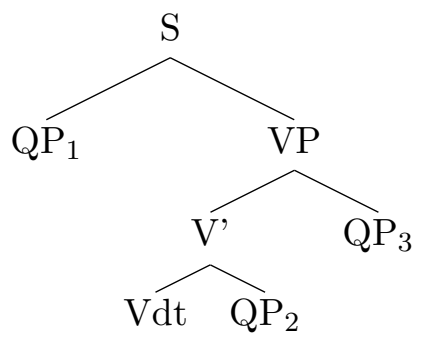

For each reading, we now define its respective shuf-operation

$Q P_{1}>Q P_{2}>Q P_{3}$

$$
\operatorname{shuf}^{1,2,3}: \mathcal{C P}\left(X_{1} \times X_{3}\right) \times \mathcal{C}\left(X_{3}\right) \rightarrow \mathcal{P C}\left(X_{1}\right)
$$

$\operatorname{shuf}^{1,2,3}\left(S_{2}, S_{3}\right)=\lambda S_{1: \mathcal{C}\left(X_{1}\right)} \cdot \operatorname{CPS}^{l}\left(\operatorname{eps}^{r} X_{1}\right)\left(S_{1}, \mathbf{C P S}^{l}\left(\operatorname{eps}_{X_{3}}^{l}\right)\left(S_{2}, S_{3}\right)\right)\left(i d_{t}\right)$ for $S_{2} \in \mathcal{C P}\left(X_{1} \times X_{3}\right)$ and $S_{3} \in \mathcal{C}\left(X_{3}\right)$.

$Q P_{3}>Q P_{2}>Q P_{1}$

$$
\operatorname{shuf}^{3,2,1}: \mathcal{C P}\left(X_{1} \times X_{3}\right) \times \mathcal{C}\left(X_{3}\right) \rightarrow \mathcal{P C}\left(X_{1}\right)
$$

$\operatorname{shuf}^{3,2,1}\left(S_{2}, S_{3}\right)=\lambda S_{1: \mathcal{C}\left(X_{1}\right)} \cdot \operatorname{CPS}^{l}\left(\operatorname{eps}^{l}{ }_{X_{1}}\right)\left(\operatorname{CPS}^{l}\left(\operatorname{eps}^{r}{ }_{X_{3}}\right)\left(S_{3}, S_{2}\right), S_{1}\right)\left(i d_{t}\right)$ for $S_{2} \in \mathcal{C P}\left(X_{1} \times X_{3}\right)$ and $S_{3} \in \mathcal{C}\left(X_{3}\right)$.

$Q P_{1}>Q P_{3}>Q P_{2}$

$$
\begin{gathered}
\operatorname{shuf}^{1,3,2}: \mathcal{C P}\left(X_{1} \times X_{3}\right) \times \mathcal{C}\left(X_{3}\right) \rightarrow \mathcal{P C}\left(X_{1}\right) \\
\operatorname{shuf}^{1,3,2}\left(S_{2}, S_{3}\right)=\lambda S_{1: \mathcal{C}\left(X_{1}\right)} \cdot \mathbf{C P S}^{l}\left(\operatorname{eps}^{r}{ }_{X_{1}}\right)\left(S_{1}, \mathbf{C P S}^{l}\left(\operatorname{eps}^{r}{ }_{X_{3}}\right)\left(S_{3}, S_{2}\right)\right)\left(i d_{t}\right)
\end{gathered}
$$


for $S_{2} \in \mathcal{C P}\left(X_{1} \times X_{3}\right)$ and $S_{3} \in \mathcal{C}\left(X_{3}\right)$.

$Q P_{2}>Q P_{3}>Q P_{1}$

$$
\operatorname{shuf}^{2,3,1}: \mathcal{C P}\left(X_{1} \times X_{3}\right) \times \mathcal{C}\left(X_{3}\right) \rightarrow \mathcal{P C}\left(X_{1}\right)
$$

$\operatorname{shuf}^{2,3,1}\left(S_{2}, S_{3}\right)=\lambda S_{1: \mathcal{C}\left(X_{1}\right)} \cdot \operatorname{CPS}^{l}\left(\operatorname{eps}^{l}{ }_{X_{1}}\right)\left(\operatorname{CPS}^{l}\left(\operatorname{eps}_{X_{3}}^{l}\right)\left(S_{2}, S_{3}\right), S_{1}\right)\left(i d_{t}\right)$ for $S_{2} \in \mathcal{C P}\left(X_{1} \times X_{3}\right)$ and $S_{3} \in \mathcal{C}\left(X_{3}\right)$.

$Q P_{3}>Q P_{1}>Q P_{2}$

$$
\operatorname{shuf}^{3,1,2}: \mathcal{C P}\left(X_{1} \times X_{3}\right) \times \mathcal{C}\left(X_{3}\right) \rightarrow \mathcal{P C}\left(X_{1}\right)
$$

$\operatorname{shuf}^{3,1,2}\left(S_{2}, S_{3}\right)=\lambda S_{1: \mathcal{C}\left(X_{1}\right)} \cdot \mathbf{C P S}^{l}\left(\mathbf{e p s}^{r} X_{3}\right)\left(S_{3}, \mathbf{C P S}^{l}\left(\operatorname{eps}^{r} X_{1}\right)\left(S_{1}, S_{2}\right)\right)\left(i d_{t}\right)$ for $S_{2} \in \mathcal{C P}\left(X_{1} \times X_{3}\right)$ and $S_{3} \in \mathcal{C}\left(X_{3}\right)$.

$Q P_{2}>Q P_{1}>Q P_{3}$

$$
\operatorname{shuf}^{2,1,3}: \mathcal{C P}\left(X_{1} \times X_{3}\right) \times \mathcal{C}\left(X_{3}\right) \rightarrow \mathcal{P C}\left(X_{1}\right)
$$

$\operatorname{shuf}^{2,1,3}\left(S_{2}, S_{3}\right)=\lambda S_{1: \mathcal{C}\left(X_{1}\right)} \cdot \operatorname{CPS}^{l}\left(\operatorname{eps}_{X_{3}}^{l}\right)\left(\operatorname{CPS}^{l}\left(\operatorname{eps}_{X_{1}}^{l}\right)\left(S_{2}, S_{1}\right), S_{3}\right)\left(i d_{t}\right)$ for $S_{2} \in \mathcal{C P}\left(X_{1} \times X_{3}\right)$ and $S_{3} \in \mathcal{C}\left(X_{3}\right)$.

The corresponding computation trees are now as follows

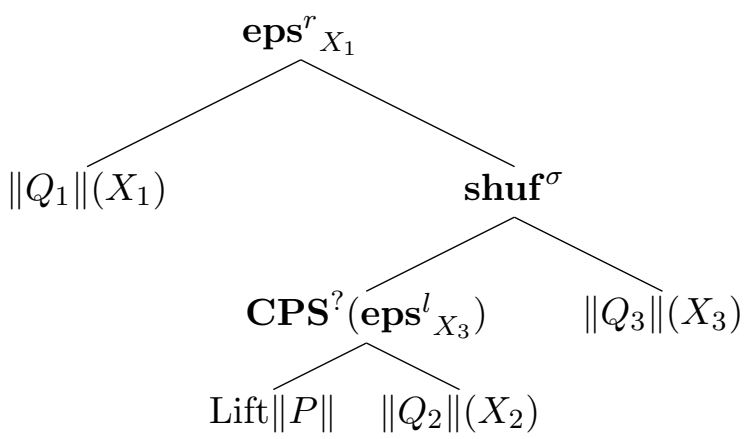

where $\sigma$ is a permutation of $\{1,2,3\}$.

Strategy $\mathbf{E}$ is both in situ and uniform, i.e., regardless of the class of sentences and the particular readings considered, it uses the same semantic operations (CPS'es, shuf'es and eps'es). Compared to Strategy D, however, it fares worse with respect to the requirement (5) that the interpretation process operate 'on the fly', i.e., the particular reading(s) of a 
sentence should be determined as late as possible. In Strategy $\mathbf{D}$, it is only the two readings missing from Strategy $\mathbf{C}$ that are predetermined by the shuf'es. In Strategy $\mathbf{E}$, it is all of the readings for sentences involving 2 or 3 QPs that are determined by the shuf'es. So between Strategy $\mathbf{D}$ and Strategy $\mathbf{E}$ there is a trade-off of gains and costs: having 'on the fly' process vs. uniformity in semantic operations. Perhaps, if it could be empirically shown that some readings take longer to process (e.g., the two readings missing from Strategy $\mathbf{C}$ ), then one could hypothesize that they also involve comparably more difficult semantic operations (i.e., shuf'es, in this case). Such empirical findings could be then taken to support Strategy D over E. Obviously, the empirical findings could be also found to support some mixed-strategy, located somewhere between Strategy D and Strategy E. One should also notice that in the in situ strategies discussed in this paper (whether or not the readings are predetermined) the arguments of the semantic operations applied are used 'on the spot', i.e., unlike in Cooper's Storage mechanism $([\underline{6})$, the arguments do not get stored and retrieved when needed.

\section{Conclusion}

Recent continuation-based semantics provide a compelling approach to quantification for providing a non-movement (in situ) analysis of quantifiers. In this paper, we have discussed three such possible continuation-based strategies, together with their relative merits and costs.

\section{Appendix: Strong Monads}

A monad on Set is a triple $(T, \eta, \mu)$, where $T:$ Set $\longrightarrow$ Set is an endofunctor, $\eta: 1_{\text {Set }} \longrightarrow T$ and $\mu: T^{2} \longrightarrow T$ are natural transformations making the following diagrams

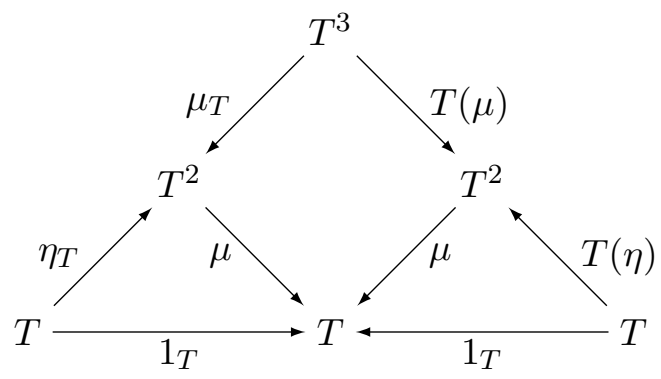


commute. $\eta$ and $\mu$ are unit and multiplication of the monad $T$, respectively.

In order to have a well behaved notion of computation, a monad has to be strong, c.f. [10], 11], [15]. Fortunately, all monads on Set are strong. Technically, we need the monad to be bi-strong as we will need to 'extend computations' both from the left and from the right. As the binary product (the only tensor that we consider in Set) is commutative, any strong monad in Set is bi-strong.

Let $(T, \eta, \mu)$ be a monad on Set. The left strength on $(T, \eta, \mu)$ is a natural transformation with components

$$
\mathbf{s t}_{X, Y}^{l}: T(X) \times Y \longrightarrow T(X \times Y)
$$

for sets $X$ and $Y$, making the diagrams

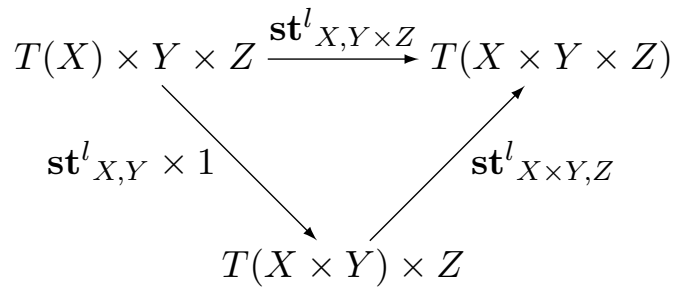

and

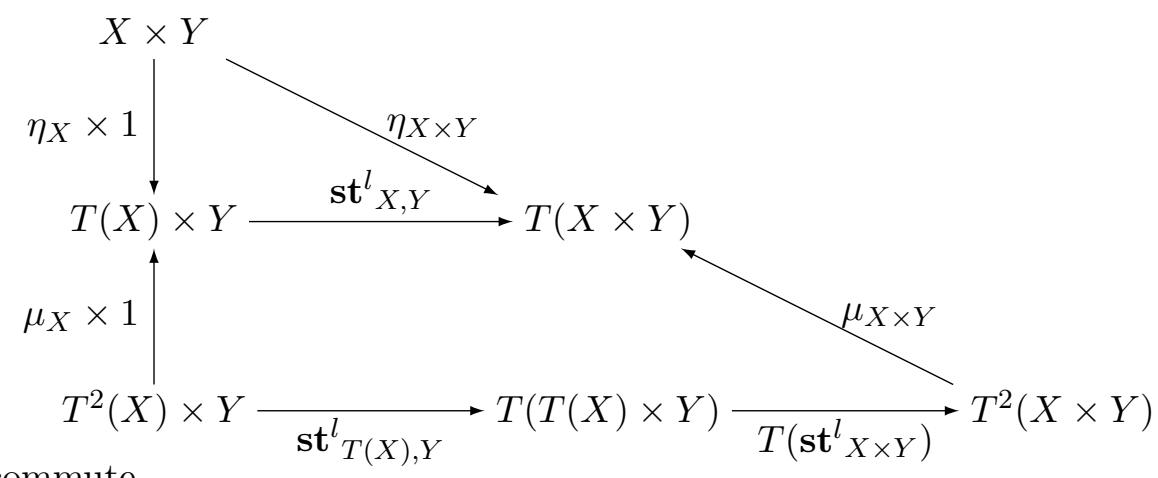

commute.

The right strength is a natural transformation with components

$$
\mathbf{s t}^{r} X, Y: X \times T(Y) \longrightarrow T(X \times Y)
$$

for sets $X$ and $Y$, making the diagrams 


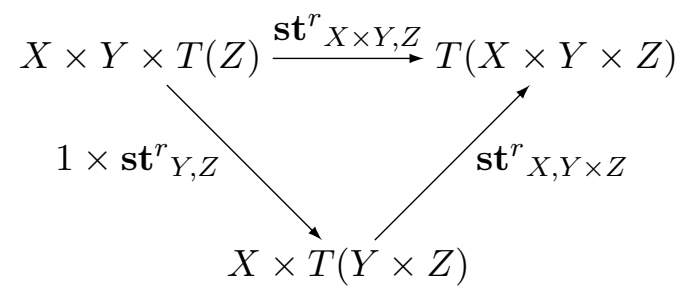

and

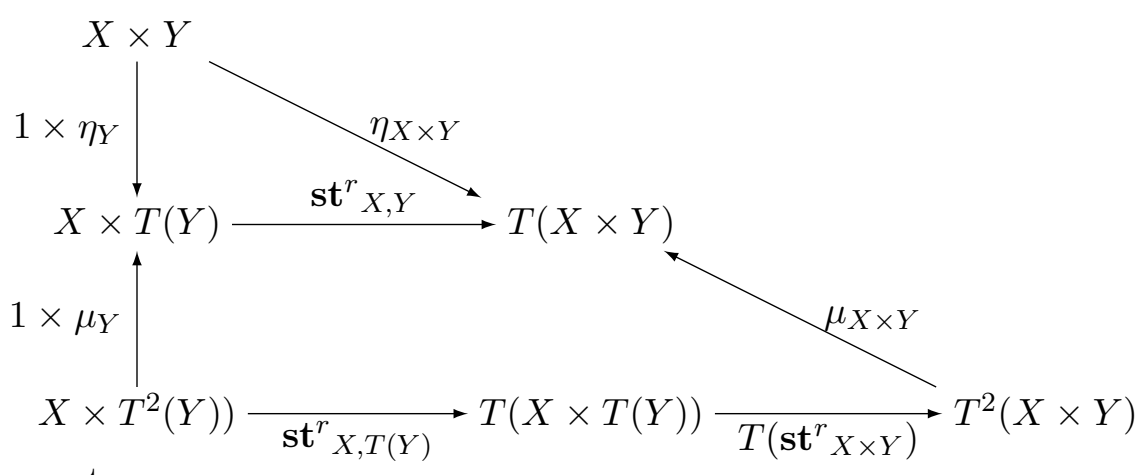

commute.

The monad $(T, \eta, \mu)$ on Set together with two natural transformations $\mathbf{s t}^{l}$ and $\mathbf{s t}^{r}$ of right and left strength is a bi-strong monad if, for any sets $X$, $Y, Z$, the square

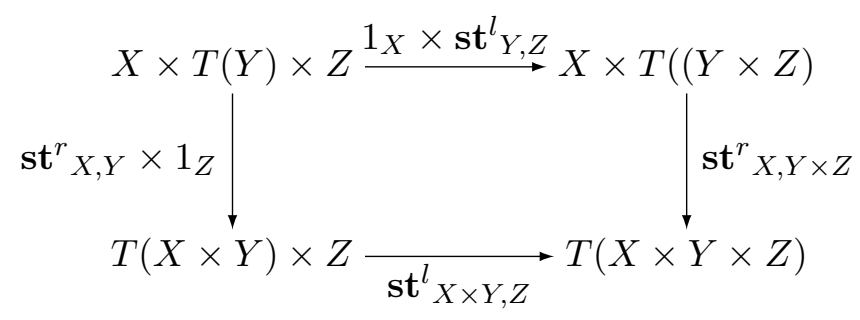

commutes.

Remarks.

1. The general definition for a strong functor on a monoidal category $(\mathcal{C}, \otimes, I, \alpha, \lambda, \rho)$ contains a yet another diagram concerning the unit. As the only tensor considered is the cartesian product, this part of the general definition is irrelevant.

2. If a monad on a symmetric monoidal category is strong, then (having, say, left strength only) one can - using symmetry - easily define 
the right strength making it a bi-strong monad. In that sense, the concept of a bi-strong monad is redundant in the symmetric monoidal categories. However, since the order does matter in the natural lan-

guage, we prefer to make both strengths explicitly given as a part of the structure.

As already mentioned, each monad $(T, \eta, \mu)$ on Set is bi-strong. We shall define the right and left strength. Fix sets $X$ and $Y$. For $x \in X$ and $y \in Y$, we have functions

$$
l_{y}: X \longrightarrow X \times Y, \text { and } \quad r_{x}: Y \longrightarrow X \times Y,
$$

such that

$$
l_{y}(x)=\langle x, y\rangle, \quad \text { and } \quad r_{x}(y)=\langle x, y\rangle .
$$

The left and right strength

st $_{X, Y}^{l}: T(X) \times Y \longrightarrow T(X \times Y) \quad$ and $\quad$ st $^{r}{ }_{X, Y}: X \times T(Y) \longrightarrow T(X \times Y)$

are given for $x \in X, s \in T(X), y \in Y$ and $t \in T(Y)$ by

$$
\mathbf{s t}_{X, Y}^{l}(s, y)=T\left(l_{y}\right)(s) \quad \text { and } \quad \mathbf{s t}_{X, Y}^{r}(x, t)=T\left(r_{x}\right)(t),
$$

respectively. We drop indices $X, Y$ when it does not lead to confusion.

One can check that the monad $(T, \eta, \mu)$ equipped with so defined natural transformations $\mathbf{s t}^{l}$ and $\mathbf{s t}^{r}$ is bi-strong.

\section{References}

[1] Barker, C.: Continuations and the nature of quantification. Natural Language Semantics 10, 211-242 (2002).

[2] Barker, C., Shan, C.c.: Continuations and Natural Language. Oxford University Press (2014).

[3] Barwise, J., Cooper, R.: Generalized Quantifiers and Natural Language. Linguistics \& Philosophy 4, 159-219 (1981).

[4] Bekki, D., Assai, K.: Representing Covert Movements by Delimited Continuations In Nakakoji, K., Murakami, Y., McCready, E. (eds.) New Frontiers in Artificial Intelligence, JSAI-isAI, LNAI 6284, 161-180, (2010). 
[5] Benthem, J.: Polyadic quantifiers. Linguistics \& Philosophy 12, 437-464 (1989).

[6] Cooper, R.: Quantification and Syntactic Structure. Dordrecht: Reidel (1983).

[7] de Groote, P.: Type raising, continuations, and classical logic. In van Rooy, R., Stokhof, M. (eds.), Proceedings of the 13th Amsterdam Colloquium, Institute for Logic, Language and Computation, Universiteit van Amsterdam 97-101 (2001).

[8] Keenan, E. L.: Unreducible n-ary quantifiers in natural language. In Grdenfors P. (ed.), Generalized Quantifier: Linguistic and Logical Approaches, Reidel Dordrecht. 109-150 (1987).

[9] Kiselyov, O., Shan, C.c.: Continuation Hierarchy and Quantifier Scope. In McCready, E., Yabushita, K., Yoshimoto, K. (eds.), Formal Approaches to Semantics and Pragmatics: Japanese and Beyond. Studies in Linguistics and Philosophy, Springer Netherlands, 105-134 (2014).

[10] Kock, A.: Monads on symmetric monoidal closed categories. Arch. Math. (Basel), 21:110 (1970).

[11] Kock, A.: Strong functors and monoidal monads. Arch. Math. (Basel), 23:113120 (1972).

[12] Lindström, P.: First-order predicate logic with generalized quantifiers. Theoria 32, 186-95.(1966).

[13] May, R.: The Grammar of Quantification. PhD dissertation, MIT (1977).

[14] May, R.: Logical Form: Its Structure and Derivation. MIT Press (1985).

[15] Moggi, E.: The notion of computation and monads. Information And Computation 93:5592 (1991).

[16] Montague, R.: The proper treatment of quantification in ordinary English. In: Thomason, R. (ed.), Formal Philosophy: Selected Papers of Richard Montague. New Haven and London: Yale University Press, 247271 (1974).

[17] Mostowski, A.: On a generalization of quantifiers. Fundamenta Mathematicae 44, 12-36 (1957). 
[18] Szabolcsi, A.: Quantification. Cambridge University Press, Cambridge (2010).

[19] Zawadowski, M.: Formalization of the feature system in terms of preorders. In Bellert, I, Feature System for Quantification Structures in Natural Language, Foris Dordrecht, 155-175 (1989).

[20] Grudzinska, J; Zawadowski, M.: Scope ambiguities, monads and strengths. arXiv:1605.03981. 